\title{
Four Measures for the Dynamics of Coalitions in Social Networks
}

\author{
Guido Boella \\ Dipartimento di Informatica, \\ University of Turin \\ C.so Svizzera 185 \\ Torino, Italy \\ guido@di.unito.it
}

\author{
Leendert van der Torre \\ CSC, University of \\ Luxembourg \\ 6 ,rue R. Coudenhove-Kalergi \\ Luxembourg, Luxembourg \\ leendert@vandertorre.com
}

\author{
Serena Villata \\ Dipartimento di Informatica, \\ University of Turin \\ C.so Svizzera 185 \\ Turin, Italy \\ villata@di.unito.it
}

\begin{abstract}
We introduce four measures for the change of coalitions in social networks. The first one measures the change of the agents in the network over time, The second one measures the change of dependencies among the agents, due to addition or removal of powers and goals of the agents. The third one measures the change in normative dependencies like obligations and prohibitions introduced by norms. The fourth one measures changes in coalitions. If one of the first three measures is high, then the fourth measure is probably high too, if the change in agents and dependencies is a cause for a change in coalitions. If the first three measures are low, but the change in coalitions is high, it is due to internal processes like violations of the coalition agreements.
\end{abstract}

\section{General Terms}

Social dependence networks, measures, coalitions' evolution.

Coalitions play a central role in social reasoning, and thus various theories have been used and developed in multiagent systems. For example, coalitional game theory has been adopted from economics and extended for multiagent systems [3], and social networks have been adopted from social sciences and modified to represent dependence networks among agents $[4,2]$. These theories differ in various ways. For example, in the former, coalitions are represented by sets of agents while in the latter, coalitions are represented by sets of dependencies. Moreover, in the former various notions of stability are defined, whereas in the latter they are not. In this paper, we address the question how to measure the evolution and the changes of a coalition over time in terms of:

Changes of the agents and dependencies. Agents enter or leave the system over time and dependencies may be added or deleted depending on the fulfillment of the related goal or the presence of the power to fulfill this goal.

Changes of the dependencies related to norms. Norms and, in particular, obligations aim to design systems regulated by norms. The norm sets a particular kind of dependency among two agents which can be deleted if the obligation is fulfilled or a new obligation can be inserted into the system to regulate its behaviour.
Internal dynamics. Changes of the coalition are expressed in terms of additions and removals of goal-based and norm-based dependencies composing the coalition, e.g., an agent is excluded from a coalition because of a malicious behaviour.

Consider the coalition of players in a soccer team. It can change because new players come in, or players retire. It can change, because agents acquire new abilities or loose abilities, e.g., they loose their form, they break a leg, or get new goals, e.g., they want to play in the national team. Concerning norms, there can be the obligation set by the trainer for a player to play in the left wing position. Concerning internal dynamics, there may be a malicious behavior of a player, e.g., he gets too many red cards since he is too aggressive and he is no longer allowed to play. The first version of dependence networks introduced by Sichman and Conte [5] describes only one type of relation, a goal-based dependency. Social structures, however, are often multiple with multiple different kinds of ties among social actors. To model a social structure more realistically, we add the normative component introducing norm-based dependencies [1]. Our model aims to distinguish and represent not only short term situations such as, e.g., a virtual meeting on Second Life but also long term situations as, e.g., the work of an office. We define two measures associated to the number of agents and the number of goal-based dependencies present in each time frame.

Definition 1 (Agents And DePEndencies Measures). Let $i$ be a time frame, $N_{i}^{\text {Agent }}$ is given by the number of agents entering the system $A_{i}^{+}$and leaving the system $A_{i}^{-}$, depending on the total number of agents $A_{i-1}$ present at time frame $i-1$ :

$$
N_{i}^{\text {Agent }}=\sum\left(\frac{A_{i}^{+}}{A_{i-1}}\right)+\sum\left(\frac{A_{i}^{-}}{A_{i-1}}\right)
$$

Let $i$ be a time frame, $N_{i}^{D e p}$ is given by the number of goal-based dependencies added to the network $D_{i}^{+}$and deleted form the network $D_{i}^{-}$, depending on the total number of goal-based dependencies $D_{i-1}$ present at time frame $i-1$ :

$$
N_{i}^{\text {Dep }}=\sum\left(\frac{D_{i}^{+}}{D_{i-1}}\right)+\sum\left(\frac{D_{i}^{-}}{D_{i-1}}\right)
$$

We distinguish, represent and measure both short term contracts, e.g., a transaction on e-Bay such as an agreement carried out between separate entities involving the exchange of goods and money, and long term contracts, e.g., the marriage contract which hopefully lasts forever. 


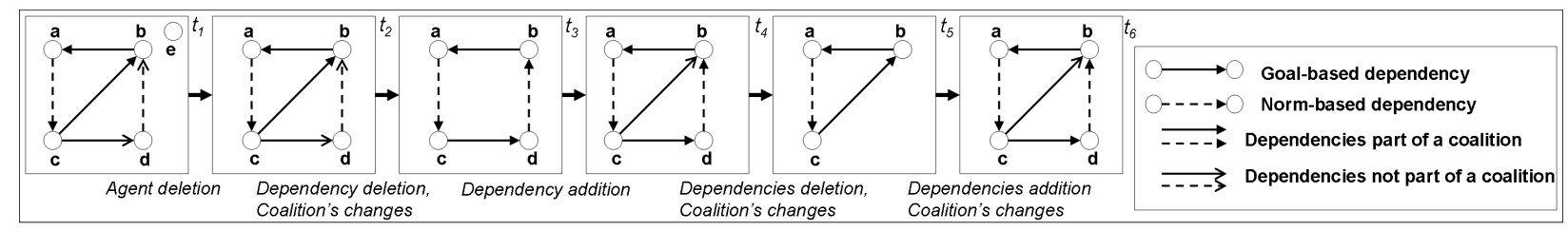

Figure 1: Coalition changes.

DEFINITION 2 (NORMS MEASURE). Let $i$ be a time frame, $N_{i}^{\text {Norm }}$ is given by the number of norm-based dependencies added to the network $O_{i}^{+}$and deleted form the network $O_{i}^{-}$, depending on the total number of norm-based dependencies $O_{i-1}$ present at time frame $i-1$ :

$$
N_{i}^{N o r m}=\sum\left(\frac{O_{i}^{+}}{O_{i-1}}\right)+\sum\left(\frac{O_{i}^{-}}{O_{i-1}}\right)
$$

We define the measure counting the number of dependencies composing the coalition in each time frame, as follows:

DEFINITION 3 (COALITIONS MEASURE). Let i be a time frame, $N_{i}^{\text {Coal }}$ is given by the number of norm-based and goal-based dependencies of a coalition added to the network $\left(D_{i}^{+}+O_{i}^{+}\right) \in C_{i}$ and deleted from the network $\left(D_{i}^{-}+O_{i}^{-}\right) \in C_{i}$ depending on the total number of norm-based and goal-based dependencies composing the coalition $\left(D_{i-1}+O_{i-1}\right) \in C_{i-1}$ at time frame $i-1$ :

$$
N_{i}^{\text {Coal }}=\sum\left(\frac{\left(D_{i}^{+}+O_{i}^{+}\right)_{C_{i}}}{\left(D_{i-1}+O_{i-1}\right)_{C_{i-1}}}\right)+\sum\left(\frac{\left(D_{i}^{-}+O_{i}^{-}\right)_{C_{i}}}{\left(D_{i-1}+O_{i-1}\right)_{C_{i-1}}}\right)
$$

Coalitions change dynamically due to rapid changes in the tasks and resource availability, and therefore relying on the initial configurations is misleading. Coalitions' evolution has to be described with the aim to maintain a history of the changes occurring to coalitions. The above measures are defined for one time moment only. We can unify these measures for a sequence of dependence networks associating to each time frame the average number of changes as follows:

DEFINITION 4 (CHANGES MEASURES). Let $i$ be a time frame of a sequence of social dependence networks, the measure of the changes' average is given by the fraction of the sum of the single measures and the number of available measures:

$$
\frac{N_{i}^{\text {Agent }}+N_{i}^{\text {Dep }}+N_{i}^{\text {Norm }}+N_{i}^{\text {Coal }}}{\text { measures }}
$$

EXAMPLE 1. In Figure 1, we present the case of six time frames visualizing the evolution of a coalition. In the first time frame, we have five agents and a coalition involving agents $a, b, c$, as shown by the dependencies composing it. There are also two norm-based dependencies and three goal-based dependencies. The passage from the first instant $t_{1}$ to the second one shows the deletion of agent $e$. From instant $t_{2}$ to instant $t_{3}$, we observe the deletion of the goal-based dependency connecting agents $c$ and $b$. Also the coalition changes and it is formed by all the four agents. From instant $t_{3}$ to instant $t_{4}$, the situation changes back to the original configuration but the coalition is fixed. From instant $t_{4}$ to instant $t_{5}$, agent $d$ disappears, a norm-based dependency is deleted and the coalition changes its actors, involving now $a, b$ and $c$. From instant $t_{5}$ to instant $t_{6}$, the situation cames back to the situation of instant $t_{4}$. Measures vary as shown in Table 1.

\begin{tabular}{|c|c|c|c|c|c|c|}
\hline & $t_{1}$ & $t_{2}$ & $t_{3}$ & $t_{4}$ & $t_{5}$ & $t_{6}$ \\
\hline$N_{i}^{\text {Agent }}$ & $0 / 5$ & $1 / 5$ & $0 / 4$ & $0 / 4$ & $1 / 4$ & $1 / 3$ \\
\hline$N_{i}^{\text {Dep }}$ & $0 / 3$ & $0 / 3$ & $1 / 3$ & $1 / 2$ & $1 / 3$ & $1 / 2$ \\
\hline$N_{i}^{\text {Norm }}$ & $0 / 2$ & $0 / 2$ & $0 / 2$ & $0 / 2$ & $1 / 2$ & $1 / 1$ \\
\hline$N_{i}^{\text {Coal }}$ & $0 / 3$ & $0 / 3$ & $3 / 3$ & $0 / 4$ & $3 / 4$ & $3 / 3$ \\
\hline Changes & 0 & 0,05 & 0,33 & 0,12 & 0,55 & 0,85 \\
\hline
\end{tabular}

Table 1: Measures of Figure 1

Thanks to the changes measure, we underline that the two time frames with the main changes in comparison with their previous time frame are $t_{3}$ and $t_{5}$, as can be supposed observing the relative figure. It can be noted that in our measures the deletion of a component increases the difference of the changes measure associated to two time frames in a row while the addition of these components causes a minor change. This behaviour is due to the relation of our measure with the game theoretical approaches for defining stability: the stability is maintained in order to avoid the breaking off of the agents from the grand coalition and form their own group.

With a more detailed model, we could make more detailed and precise distinctions between the four kinds of changes. However, often we only have the given information, for example in systems' design, and we already would like to do the analysis on these models. Concerning future work, although stability in our model can be identified intuitively in the absence of coalitions' changes, it is necessary to provide a formal definition of this notion and to associate it a measure able to represent it.

\section{REFERENCES}

[1] Guido Boella, Leendert van der Torre, and Serena Villata. Changing institutional goals and beliefs of autonomous agents. In PRIMA' 08. LNCS, Springer.

[2] L. Sauro. Formalizing admissibility criteria in coalition formation among goal directed agents. $\mathrm{PhD}$ thesis, University of Turin, 2005.

[3] Y. Shoham and K. Leyton-Brown. Multiagent Systems: Algorithmic, Game-Theoretic, and Logical Foundations. Cambridge University Press, 2008.

[4] J. S. Sichman. Depint: Dependence-based coalition formation in an open multi-agent scenario. Artificial Societies and Social Simulation, 1(2), 1998.

[5] J. S. Sichman and R. Conte. Multi-agent dependence by dependence graphs. In $A A M A S^{\prime}$ '02, pages 483-490, 2002. 